\title{
Ultra-Cold Methods for Polarized Atomic Hydrogen
}

\author{
V.G. Luppov', J.D. Arnold', B.B. Blinov', M.A. Bychkov ${ }^{1,3}$, \\ S.E. Gladycheva ${ }^{1}$, A.D. Krisch ${ }^{1}$, A.M.T. Lin ${ }^{1}$, R.S. Raymond ${ }^{\prime}$, \\ V.V. Fimushkin ${ }^{2}$, V.V. Mochalov ${ }^{3}$, P.A. Semenov ${ }^{3}$ \\ (I) Randall Lab. of Physics, University of Michigan, Ann Arbor, MI 48109-1120, USA \\ (2) Joint Institute for Nuclear Research, Dubna, RU-141980, Russia \\ (3) Institute for High Energy Physics, Protvino, RU-142284, Russia
}

\begin{abstract}
Using the ultra-cold electron-spin-polarized atomic hydrogen technique, one can produce a slow monochromatic beam for use as a polarized jet target. We will first review the development of the ultra-cold technique and then discuss the recent progress on Michigan's Mark-II ultra-cold proton-spin-polarized hydrogen jet target.
\end{abstract}

\section{INTRODUCTION}

Electron-spin-polarized hydrogen atoms in the lower hyperfine states $|3\rangle$ and 14$\rangle$ can be stabilized for a long time in a magnetic field above $5 \mathrm{~T}$ at a temperature below $500 \mathrm{mK}$ (see Fig.1). Under these conditions, the electron-spin magnetic energy difference between states with the electron spin "up" and "down" $\left(2 \mu_{e} B\right)$ is much larger than the thermal energy $(\mathrm{kT})$; therefore, only the two lowest states are significantly populated. For a magnetic field of $8 \mathrm{~T}$ and an atomic hydrogen temperature of $300 \mathrm{mK}$, the ratio of electron-spin-down to electron-spin-up states is $\exp \left(2 \mu_{\mathrm{e}} \mathrm{B} / \mathrm{kT}\right)=3.610^{15}$.

The first successful long-term stabilization of atomic hydrogen gas (1) used a high magnetic field and an ultra-cold cell coated with superfluid ${ }^{4} \mathrm{He}$ to suppress surface depolarization and recombination into molecular hydrogen. Atomic hydrogen densities of up to $310^{17}$ atoms $\mathrm{cm}^{-3}$ have been achieved using this technique (2). It was then proposed to use high density stabilized ultra-cold electron-spin-polarized atomic hydrogen in polarized sources and targets $(3,4,5)$. We will discuss the general methods and the main results of the ultra-cold technique. 


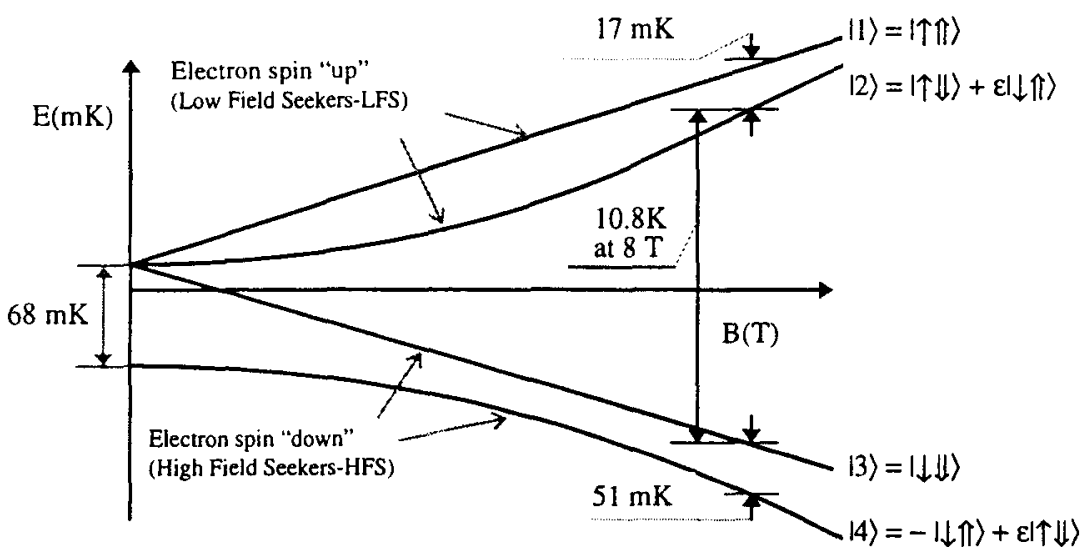

FIGURE 1. Hyperfine energy level diagram of a hydrogen atom in a magnetic field B. (Not to scale)

\section{MICROWAVE EXTRACTION OF STABILIZED ELECTRON- SPIN-POLARIZED ATOMIC HYDROGEN}

One early proposal involved electron-spin resonance (ESR) pumping and the subsequent extraction of stored hydrogen atoms from a magnetic bottle $(3,4)$. The atoms pumped from a lower hyperfine state $|4\rangle$ or $|3\rangle$ to an upper state $|1\rangle$ or $|2\rangle$, were focused and driven by a magnetic field gradient into the low magnetic field region, where they formed a slow beam with a small divergence and a small velocity spread. Several authors studied spin-polarized atomic hydrogen ESR pumping $(6,7)$ and one observed atoms ejected from a microwave cavity into a closed storage cell (8). The first electron-spin-polarized atomic hydrogen beam was formed by microwave extraction around 1991 (9). In this experiment the ESR transition at a $5 \mathrm{~T}$ field was driven by up to $60 \mathrm{~mW}$ of $140 \mathrm{GHz}$ microwave radiation in a storage cell, which was produced by a Varian $20 \mathrm{~W}$ Extended Interaction Oscillator.

Atomic hydrogen was later microwave extracted from a $7.6 \mathrm{~T}$ magnetic field (10). The external magnetic field and the $300 \mathrm{mK}$ stabilization cell, of $36 \mathrm{~cm}$ length and $2.5 \mathrm{~cm}$ diameter, are shown in Fig. 2. Atomic hydrogen at $20 \mathrm{~K}$ was fed into this cell, which was coated with superfluid ${ }^{4} \mathrm{He}$; the atoms passed through baffles which cooled them to $300 \mathrm{mK}$; they were then electron-spinseparated by the magnetic field gradient. The electron-spin-up atoms were repelled by the gradient and did not enter the cell, while the electron-spin-down atoms were attracted into the central cell volume and stabilized. The spin-up atoms could either recombine or depolarize into spin-down atoms and then enter the cell. 


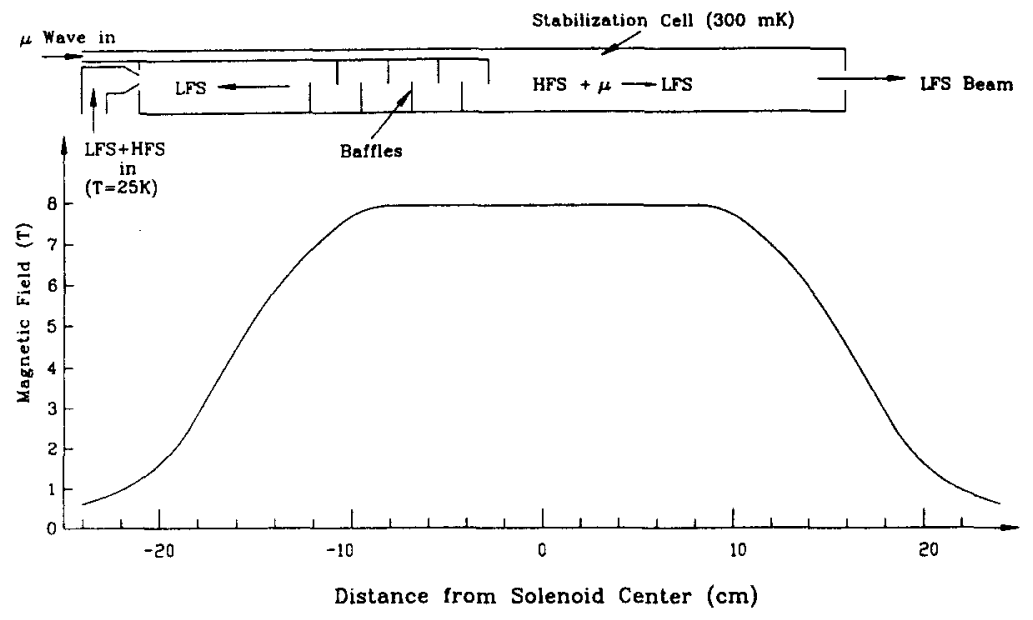

FIGURE 2. The stabilization cell used in $(10,11)$ is displayed relative to the solenoid field.

The ESR transition was driven at the resonance frequency, by $213 \mathrm{GHz}$ microwaves, produced by a $71 \mathrm{GHz}$ Klystron and a microwave tripler; however, the maximum microwave power available at the storage cell was only $5 \mathrm{~mW}$.

We later studied the same microwave-driven extraction of stabilized electronspin-polarized atomic hydrogen with a $212 \mathrm{GHz}$ microwave source of $2 \mathrm{~W}$ (11). The extracted beam was focused by a sextupole. We observed the extracted atomic hydrogen beam using both a compression tube detector and a thermal ring detector at $5 \mathrm{~K}$. Fig. 3 shows a typical thermal detector signal obtained by ramping the magnetic field, with $9 \mathrm{~mW}$ of microwave power going into the stabilization cell. The two peaks, corresponding to the $|4\rangle \rightarrow|1\rangle$ and $|3\rangle \rightarrow|2\rangle$ transitions, are separated by $510 \pm 20 \mathrm{G}$, which is in good agreement with the calculated hyperfine splitting of $507 \mathrm{G}$ or $68 \mathrm{mK}$. A typical measured compression tube signal is plotted against the microwave power at the stabilization cell in Fig. 4. The maximum observed continuous atomic hydrogen flow into the compression tube of $710^{14}$ atoms $\sec ^{-1}$ corresponded to a jet density of about $510^{10}$ atoms $\mathrm{cm}^{-3}$.

Note that the proton polarization of the extracted beam is near zero. This occurs because the stabilization cell atoms are almost equally in the $|1\rangle,|2\rangle,|3\rangle$ and $|4\rangle$ states, which is due to spin-exchange processes, such as $|4\rangle+|2\rangle \leftrightarrow|3\rangle+|1\rangle$, having very large rates (6). 


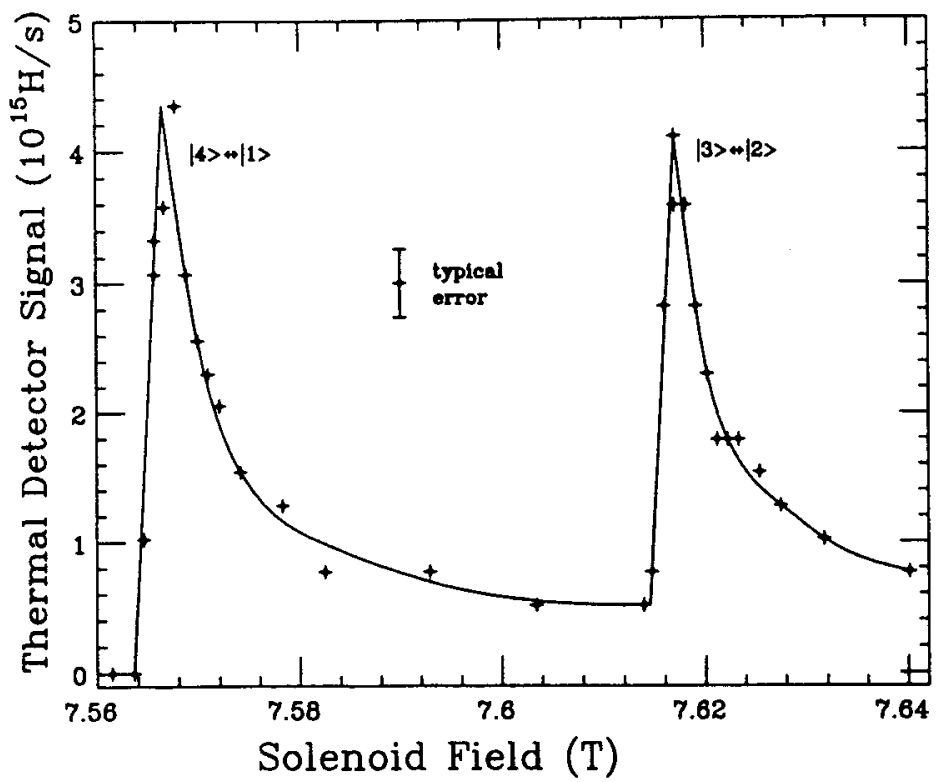

FIGURE 3. The thermal detector signal is plotted versus the ramped magnetic field (11).

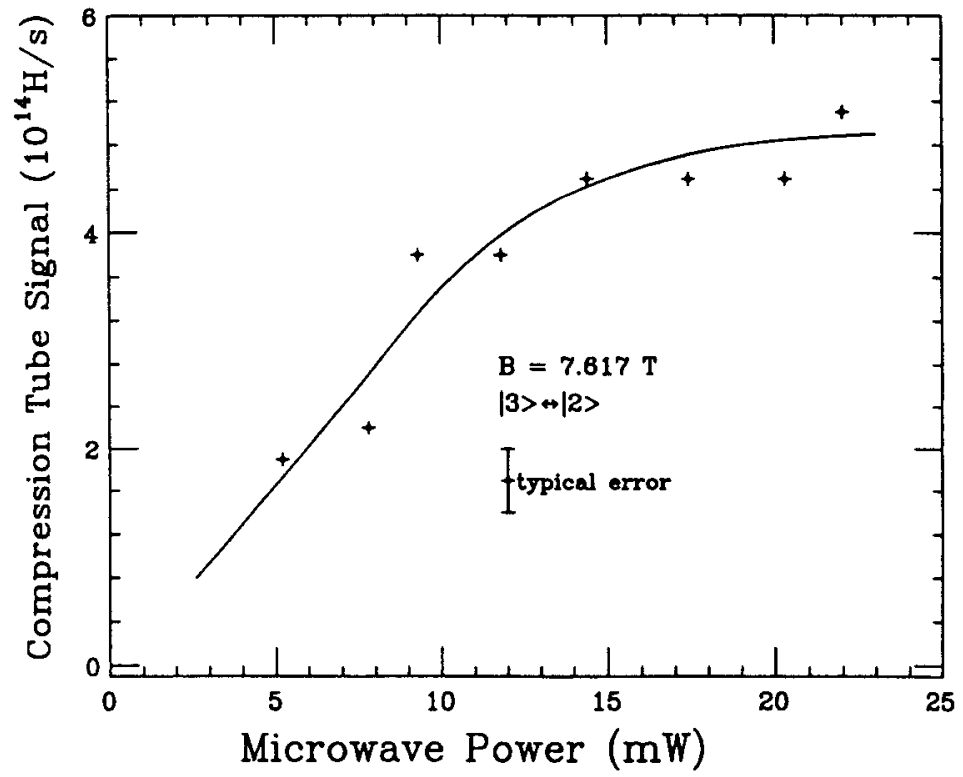

FIGURE 4. The compression tube signal is plotted versus the microwave power into the stabilization cell (11). 


\section{MAGNETIC-FIELD-GRADIENT ELECTRON-SPIN- SEPARATION OF ULTRA-COLD ATOMIC HYDROGEN}

It was proposed (5) to use the steep magnetic field gradient itself to separate the ultra-cold hydrogen atoms of different electron-spin states. The Michigan prototype jet was then modified to study the continuous production of ultra-cold electron-spin-polarized hydrogen atoms (10), as shown in Fig. 5.

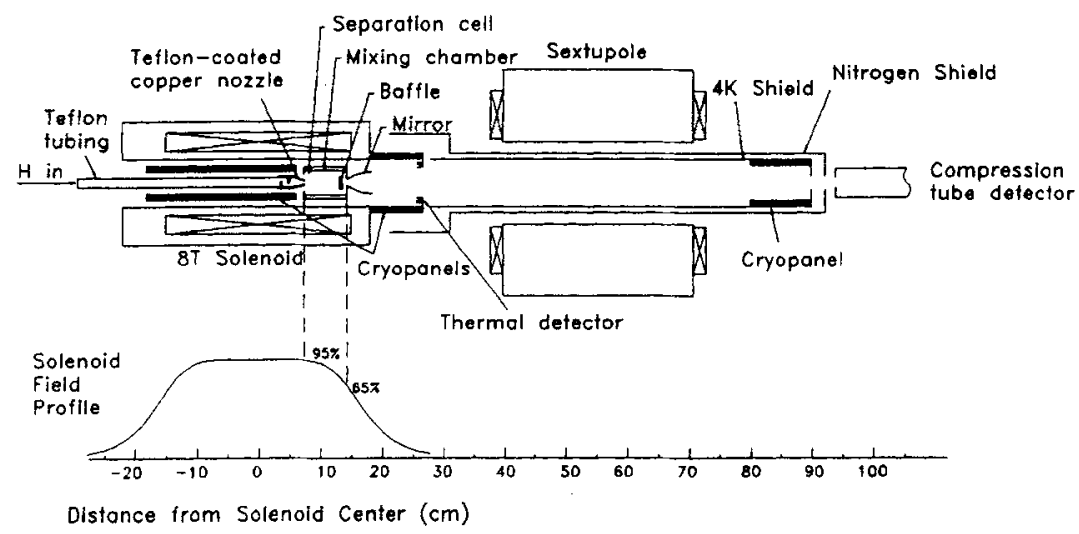

FIGURE 5. The Michigan Prototype ultra-cold electron-spin-polarized atomic hydrogen jet.

Atomic hydrogen was guided into the $300 \mathrm{mK}$ ultra-cold separation cell through a Teflon-coated copper nozzle held at about $20 \mathrm{~K}$. The cell was covered with a superfluid ${ }^{4} \mathrm{He}$ film to suppress the atomic hydrogen surface recombination. The cell's entrance and exit apertures were respectively located at $95 \%$ and $65 \%$ of the central $8 \mathrm{~T}$ solenoid field.

After the hydrogen atoms were thermalized by collisions with the cell surface, the magnetic field gradient separated the atoms according to their electron-spin states. The atoms in the two lowest hyperfine states in Fig. 1 (high field seekers) were attracted toward the high field region. Most of these atoms eventually escaped from the cell through an annular gap around the entrance nozzle; then they recombined on bare surfaces and were pumped away by cryopanels. The atoms in the two higher hyperfine states (low field seekers) were repelled toward the low field region, where they exited through a 5-mm-diam aperture. After emerging from the aperture, these electron-spin-polarized atoms were magnetically accelerated by the remaining field gradient. About $310^{15}$ electron- 
spin-polarized atoms per second reached the detectors; however, note that the sextupole had a small acceptance; thus, only $3 \%$ of the effused atoms reached the compression tube. This observed flux corresponded to a target density of about $1.310^{11}$ atoms $\mathrm{cm}^{-3}$.

A pulsed ultra-cold polarized atomic hydrogen source using the original magnetic field gradient separation idea (5) is under development in Dubna. They have extracted a pulsed atomic beam with a $300 \mathrm{~ms}$ duration and a $0.25 \mathrm{~Hz}$ repetition frequency (12).

\section{FOCUSING AN ATOMIC HYDROGEN BEAM WITH A HELIUM-FILM-COATED QUASIPARABOLIC MIRROR}

The quantum reflection of cold hydrogen atoms from a superfluid-helium-filmcovered surface was first demonstrated by Berkhout $e t$ al. (13). They measured about $80 \%$ specular reflectivity for normal incidence on a hemispherical opticalquality concave quartz mirror coated with a $100 \mathrm{mK}{ }^{4} \mathrm{He}$ film. The quantum reflection occurs because hydrogen atoms are light and interact very weakly with the helium surface.

The first formation of an external beam of ultracold electron-spin-polarized hydrogen atoms, using a highly polished quasiparabolic copper mirror coated with ${ }^{4} \mathrm{He}$ film, was reported in 1993 (14). The Michigan prototype jet (10), using the magnetic-field-gradient electron-spin-separation method, was used to perform these measurements. The mirror was located in the gradient of an $8 \mathrm{~T}$ solenoid magnet and mounted on an ultra-cold separation cell at $350 \mathrm{mK}$ (see Fig. 5). After the formation by the mirror, the beam was focused with a sextupole magnet. The mirror, which was especially designed for operation in the magnetic field gradient of our solenoid, increased the focused beam intensity by a factor of about 7.5 for a fixed atomic hydrogen feed rate. The $3.710^{15}$ ultra-cold low-velocity atoms per second, focused into the compression tube, corresponded to a jet target density of about $310^{11}$ atoms $\mathrm{cm}^{-3}$. Note that this kind of mirror could be used for any ultracold atomic hydrogen beam, including a beam obtained with microwave extraction.

\section{MARK-II ULTRA-COLD POLARIZED HYDROGEN JET.}

At the University of Michigan High Energy Spin Physics Lab, we are developing an ultra-cold high-density jet target of proton-spin-polarized hydrogen atoms. The Mark- $\Pi$ jet, which is shown in Fig. 6, uses the magnetic-field-gradient electron-spin-separation method described above. 


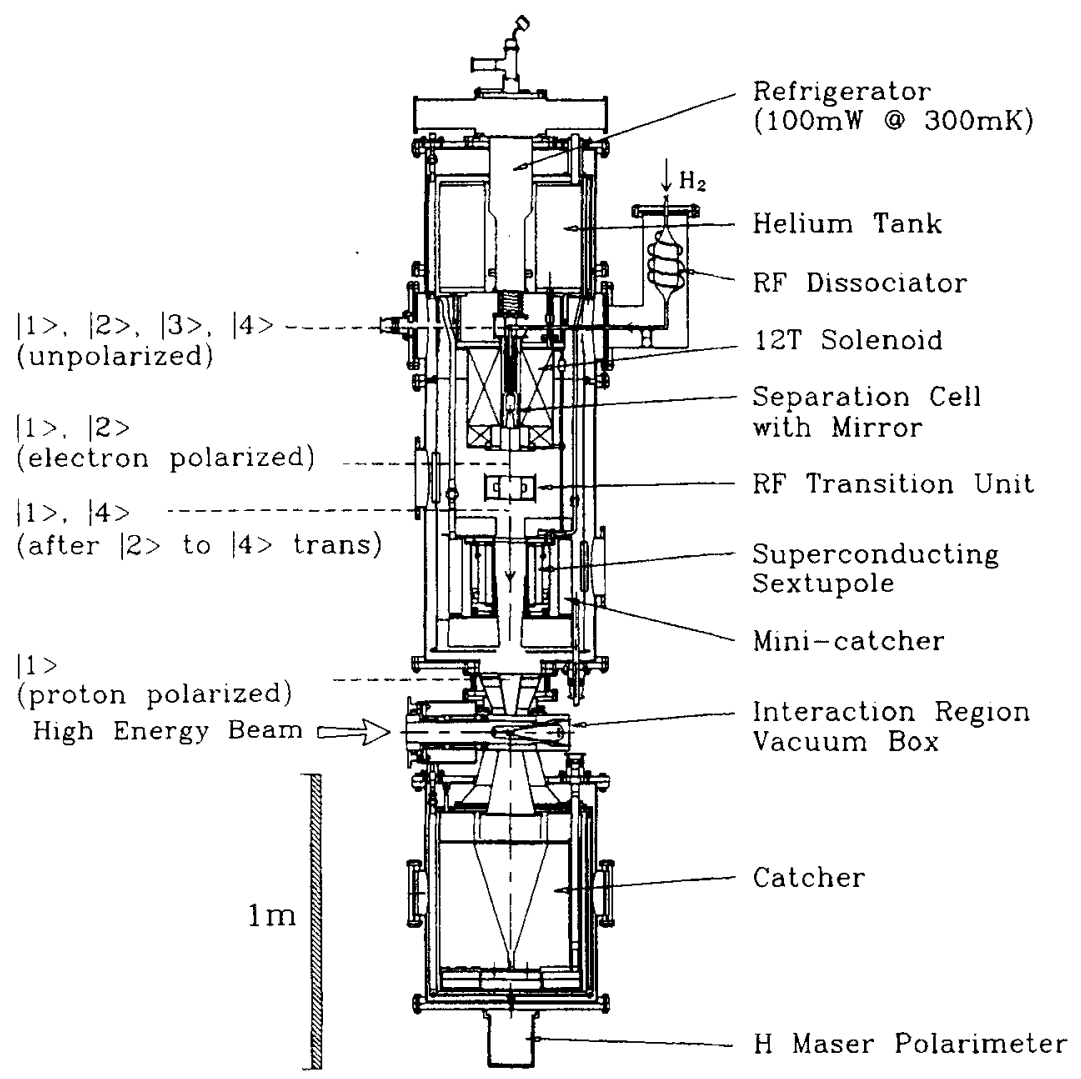

FIGURE 6. Layout of the Mark-II ultra-cold jet

Atomic hydrogen is first produced in a room-temperature if dissociator and then guided through a Teflon transport tube and a Teflon-coated copper nozzle into the $0.3 \mathrm{~K}$ separation cell, which is coated with superfluid ${ }^{4} \mathrm{He}$. The double walls of the cell form the mixing chamber of the dilution refrigerator. The cell's entrance and exit apertures are respectively located at about $95 \%$ and $50 \%$ of the superconducting solenoid's $12 \mathrm{~T}$ central magnetic field. After the hydrogen atoms are thermalized by collisions with the cell surface, the magnetic field gradient separates the atoms according to their electron-spin states. The two higher hyperfine state atoms $|1\rangle$ and 12$\rangle$ are repelled toward the low field region and effuse from the exit aperture, forming an electron-spin-polarized beam. We plan to use a focusing mirror, with a polished surface covered with superfluid ${ }^{4} \mathrm{He}$, similar to the prototype mirror (14). The mirror should significantly increase the jet density. After the if transition unit changes the state 12$\rangle$ atoms into state 14$\rangle$ 
atoms, a superconducting sextupole focuses the state 11) atoms into the interaction region while defocusing the state 14) atoms, which are then cryopumped. The proton-spin polarized beam then passes through the interaction region and is caught below by the cryopumping catcher. A hydrogen maser polarimeter is placed below the catcher to monitor the proton polarization.

The ultra-cold method has several advantages compared to other methods:

- The ultra-cold beam divergence is much less. As shown in Fig 7a, for molecular effusion from a thin-walled aperture, the atoms' angular distribution is proportional to $\cos \theta$, where $\theta$ is measured normal to the aperture. The ultracold atoms are then accelerated by a longitudinal magnetic field, which turns their trajectories toward the axis and narrows their angular distribution. MonteCarlo simulations of the angular distributions before and after the acceleration, respectively, are shown in Fig. $7 \mathrm{a}$ and $7 \mathrm{~b}$ for the $12 \mathrm{~T}$ Mark- $\Pi$ with the separation cell exit aperture at $6 \mathrm{~T}$ and a field gradient of about $1 \mathrm{~T} \mathrm{~cm}^{-1}$. As shown in Fig. 7c, a focusing quasiparabolic mirror should further decrease the beam divergence and thus significantly increase the jet density.

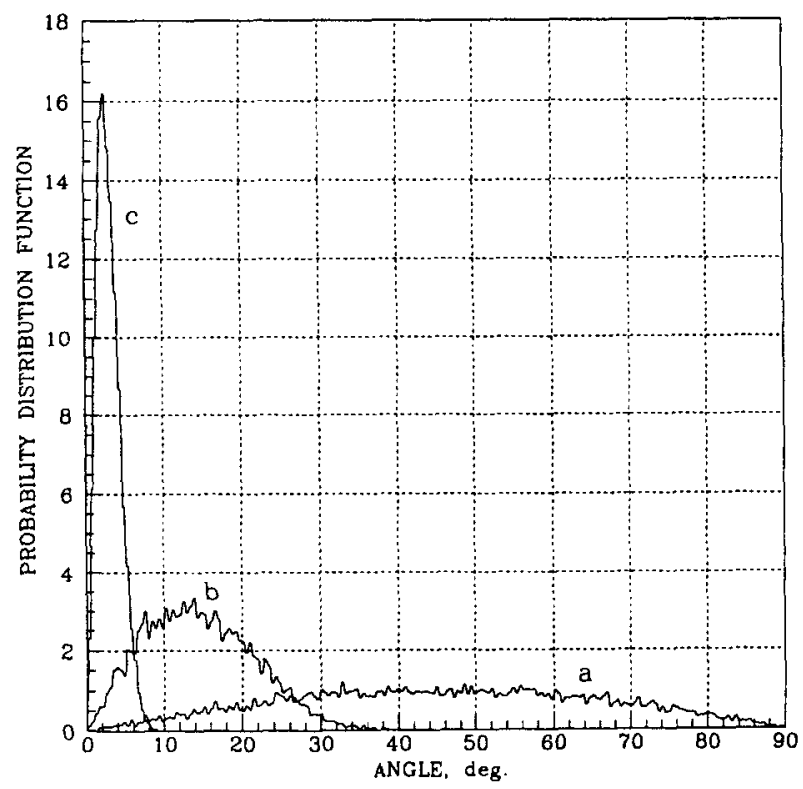

FIGURE 7. Calculated angular distributions of the $|1\rangle$ and $|2\rangle$ states: a) emerging from the cell, b) after acceleration by the magnetic field gradient with no mirror, and c) after acceleration with a quasi-parabolic mirror. 
- The ultra-cold beam is much more monochromatic due to the large magnetic-field-gradient acceleration. This monochromaticity allows the beam to be focused into the interaction region with much higher efficiency. The simulations indicate that about $45 \%$ of the beam emerging from the separation cell should be focused into a $1 \mathrm{~cm} \times 2 \mathrm{~cm}$ target region.

- A free jet's density varies inversely with the atoms' velocity. Since the ultracold atoms' average kinetic energy corresponds to about $4 \mathrm{~K}$, the target density should be 4.5 times higher than at liquid nitrogen temperature of $80 \mathrm{~K}$.

Most of the Mark-II parts have been fabricated and successfully tested. This hardware includes: a $12 \mathrm{~T}$ superconducting solenoid; a dilution refrigerator with a cooling power of about $50 \mathrm{~mW}$ at $300 \mathrm{mK}$; its pumping system; a 20-cm-long superconducting sextupole magnet with $10.5 \mathrm{~cm}$ diameter bore iron poles; a cryocondensation pump with a measured pumping speed of about $1.210^{7}$ liters $\mathrm{sec}^{-1}$ which is $4.210^{26}$ atoms Torr ${ }^{-1} \sec ^{-1}(15)$; a hydrogen maser polarimeter capable of monitoring the polarization to about $\pm 2 \%$ precision in a few minutes; and the Mark-II computer-controlled instrumentation.

The electron polarization will be converted into proton polarization by adiabatic passage through an if transition unit with a novel ring dielectric resonator that accepts a 6-cm-diameter beam. A room temperature prototype if unit was recently tested; its measured transition efficiency was about $95 \%$ (16). We recently started the first beam tests of Mark-II. Fig. 8 shows the measured atomic hydrogen transport line efficiency plotted against the nozzle temperature. This efficiency includes both the dissociation efficiency and the recombination losses on the Teflon transport tube and the nozzle.

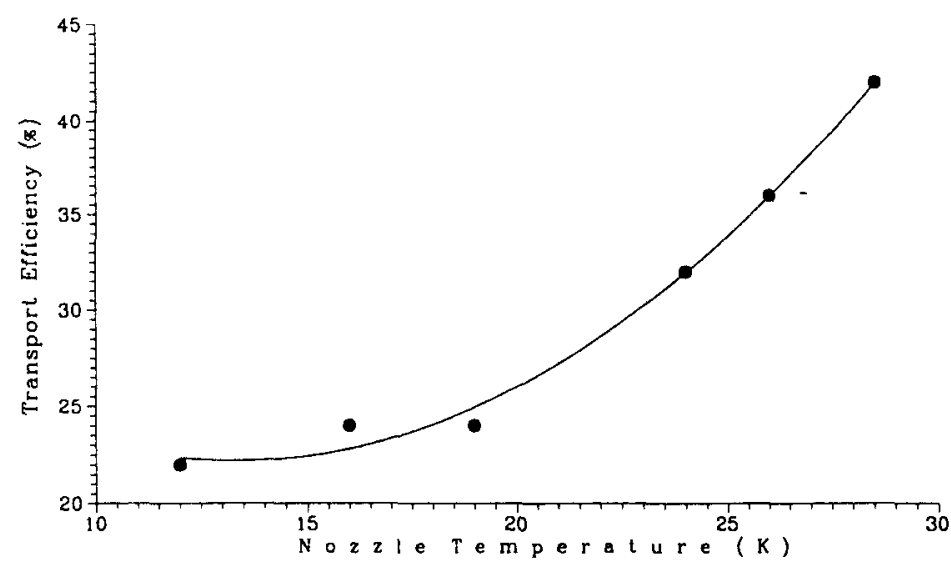

FIGURE 8. Atomic hydrogen transport efficiency versus the Teflon-coated copper nozzle's temperature. 
Extrapolating our prototype jet experimental results (14), we expect to achieve a Mark-II target thickness of about $10^{13}$ atoms $\mathrm{cm}^{-2}$ with a 2-cm-thick jet crossing the accelerator beam.

\section{ACKNOWLEDGEMENTS}

We would like to thank Prof. D. Kleppner (MIT) for helpful discussions. This work was supported by the U.S. Department of Energy.

\section{REFERENCES}

1. Silvera, I.F., and Walraven, J.T.M., Phys.Rev. Lett. 44, 164 (1980).

2. For a review of the physics of spin stabilized atomic hydrogen, see Silvera, I.F., and Walraven, J.T.M., in Progress in Low Temperature Physics (Elsevier Science Publisher B.V., Amsterdam), Vol. X, 139-370 (1986).

3. Niinikoski, T.O., in Proceedings of the International Symposium on High-Energy Physics with Polarized Beams and Polarized Targets, Lausanne, 1980 (Birkhauser EXS 38, Basle, 191 (1981)).

4. Kleppner, D., in Proceedings of the Workshop on Polarized Proton lon Sources, Ann Arbor, 1981 (AIP Conference Proceedings, No. 80, 111 (1982)).

5. Mertig, M., Levkovich, A.V., Luppov, V.G., and Pilipenko, Yu.K., in Proceedings of the 9-th International Symposium on High-Energy Spin Physics, Bonn, 1990 (Springer-Verlag, 164 (1991)).

6. Niinikoski, T.O., Penttila, S., Rieubland, J.-M., and Rijllart, A., in Proceedings of the Workshop on High Intensity Polarized Proton Ion Sources, TRIUMF, Vancouver, 1983 (AIP Conference Proceedings, No.117, 139 (1984)).

7. Matthey, A.P.M., van Zwol, J., Walraven, J.T.M., and Silvera, I.F., Phys. Rev. B 37, 4831 (1988).

8. Hurlimann, M.D., Hardy, W.N., Berlinsky, A.J., and Cline, R.W., Phys. Rev. A 34, 1605 (1986).

9. Roser, T., et al., Nucl. Instr. and Methods, A 301, 42 (1991).

10. Kaufman, W.A., Roser, T., and Vuaridel B., Nucl. Instr. and Methods, A 335, 17 (1993).

11. Kaufman, W.A., Krisch, A.D., Luppov, V.G., and Raymond, R.S., High power microwave extraction of stabilized electron-spin polarized atomic hydrogen, to be submitted to $\mathrm{Nucl}$. Instr, and Methods.

12. Fimushkin, V.V., and Pilipenko, Yu.K., in Proceedings of the 6th International Workshop on High Energy Spin Physics, Protvino, 1995 (Institute for High Energy Physics, 167 (1996)).

13. Berkhout, J.J., et al., Phys. Rev. Lett. 63, 1689 (1989).

14. Luppov, V.G., Kaufman, W.A., Hill, K.M., Raymond, R.S., and Krisch, A.D., Phys. Rev. Lett. 71, 2405 (1993).

15. Arnold, J.D., et al., Nucl. Instr. and Methods, A 391, 398 (1997)

16. Raymond, R.S., Tests of a Prototype Large-Bore, Low Power $|2\rangle$ to $|4\rangle$ RF Transition Unit, in Proceedings of this Workshop. 